\title{
CYCLIC LARGE SETS OF STEINER TRIPLE SYSTEMS OF ORDER 15
}

\author{
KEVIN T. PHELPS
}

\begin{abstract}
We examine a class of large sets of Steiner triple systems of order 15 having an automorphism consisting of two fixed points and a 13-cycle. We exhibit all members of this class: there are 256 nonisomorphic systems. We examined these members for initial configurations which could lead to a large set of Steiner quadruple systems of order 16 and established that no large set exists having three fixed points and a 13-cycle.
\end{abstract}

\section{INTRODUCTION}

A Steiner triple system of order $v$, briefly $S T S(v)$, is a pair $(V, B)$, where $V$ is a $v$-set and $B$ is a set of triples of $V$ such that every 2-subset of $V$ is contained in exactly one triple. It is well known that an $S T S(v)$ exists if and only if $v \equiv 1$ or $3 \bmod 6$.

Let $K_{v}^{3}$ denote the set of all 3-subsets of a set, $V$ (i.e., complete 3-uniform hypergraph); a partition of $K_{v}^{3}$ into $v-2$ sets $B_{1}, B_{2}, \ldots, B_{v-2}$ such that, for each $i=1,2, \ldots, v-2,\left(V, B_{i}\right)$ is an $\operatorname{STS}(v)$, is said to be a large set of $S T S(v)$. In a series of papers, Lu Jia $\mathrm{Xi}$ [8-14] established the existence of a large set of $S T S(v)$ for all $v \equiv 1$ or $3 \bmod 6, v \geq 9$, with a handful of possible exceptions (or a possible handful of exceptions, see the unfinished manuscript [14]). With the existence question essentially settled, one considers next the enumeration problem.

Large sets of $\operatorname{STS}(7)$ do not exist. There exist two large sets of $\operatorname{STS}(9)$ and at least two large sets of $S T S(13)$ (cf. [15]). One of the first constructions of large sets of $S T S(v+2)$ produced sets invariant under the cycle group of order $v$; that is to say, there is an automorphism consisting of two fixed points and a $v$-cycle which permute the $v$ disjoint $\operatorname{STS}(v+2)$ among themselves. Such systems exist infinitely often [16], however it is still open as to whether they exist for all admissible orders. We will refer to large sets of $S T S(v+2)$ having this automorphism as cyclic large sets of $\operatorname{STS}(v+2)$.

Cyclic large sets of $S T S(v+2)$ have been completely enumerated for $v=$ 5,7 , and 11 ([6]; cf. [15]). We have enumerated all nonisomorphic systems for $v=13$, i.e., all cyclic large sets of $S T S(15)$, and found 256 such systems.

Received May 8, 1989.

1980 Mathematics Subject Classification (1985 Revision). Primary 05B05, 05B07, $05 \mathrm{~B} 30$.

This research was supported by NSF grant DMS-8802261 and by NSA grant MDS-904-89-H2016. 
A second related problem is that of (cyclic) large sets of Steiner quadruple systems of order $v$. Here we ask for a partition of $K_{v}^{4}$, the set of all 4-subsets of a $v$-set, into $v-3$ disjoint sets such that each set is the set of blocks of a Steiner quadruple system; that is, any two 4-subsets contained in the same part of the partition have at most two points in common.

Similarly, a cyclic large set of quadruple systems is one having an automorphism consisting of three fixed points and a $v-3$ cycle. No large set of Steiner quadruple systems has been found. It is known that none exists for $n=8$ and 10 [7] and that no cyclic large set exists on 14 points [6]. We also considered cyclic large sets of Steiner quadruple systems of order 16 and established that none exists.

\section{CyClic large SETS OF $S T S(15)$}

Steiner triple systems of order 15 were first enumerated by Cole, Cummings, and White [2] and enumerated again with the aid of a computer by Hall and Swift [5]. There are 80 nonisomorphic STS(15); of these, 63 occur in some cyclic large set. In the appendix (see the Supplements section at the end of this issue), we list for each of the 80 different triple systems their cyclic index vector and the solutions (if any) in which they occur. The numbering of the triple systems refers to the standard numbering found in [15].

Prior to this, several cyclic large sets of $S T S(15)$ had been constructed by Denniston [3, 4], one of which was "doubly resolvable". This means that each of the disjoint triple systems could be further partitioned into parallel classes, or 1-factors. There are four $S T S(15)$ which are resolvable in this sense, and these are indicated by $(*)$ in the summary listing. Note that the doubly resolvable solution discovered by Denniston is unique.

It is easy to see, using Bays' Theorem [1], that any two cyclic large sets of $\operatorname{STS}(15)$ which are isomorphic must be multiplier isomorphic. Hence, a simple isomorphism rejection routine was easy to implement as part of the search program.

The 3-subsets of $Z_{13}$ (the integers mod 13) fall into orbits of length 13 under the action of $\left\langle Z_{13},+\right\rangle$. The search for cyclic solutions involved finding one representative of each orbit such that these representatives would form a partial triple system. The leave, or set of uncovered pairs, of this partial triple system on $\{0,1, \ldots, 12\}$ must consist of one isolated vertex and 12 vertices having degree 2 . Triples containing the fixed points 13,14 are then formed by joining these points to an appropriate pair in the leave graph. Of course this is not always possible. The isolated point is joined with the pair $\{13,14\}$; the rest of the leave graph is partitioned into two disjoint 1 -factors such that in each 1 -factor every difference $(\bmod 13)$ is in some edge.

Thus, associated with each fixed point is a "starter" or cyclic 1-factorization. Again isomorphic "starters" must be multiplier isomorphic. We find 14 nonisomorphic starters occurring among our solutions. We list these in the supplement. 
In principle, the leave graph could admit different factorizations; however, in all but one solution the factorization was unique. This exception, solution \#130, admits an alternate factorization which gives solution \#255. It is not included in the listing.

Finally, the listing of all solutions starts with the list of nonisomorphic "starter" followed by a set of representatives of the orbits of triples (mod 13). For each representative triple $\{x, y, z\}$ we have $x+y+z \equiv 0(\bmod 13)$.

Each solution has the solution number, followed by the two 1-factorizations associated with the fixed points along with the multipliers which map these 1factors (listed above) onto the leave graph. Thus, solution \#0 has 1 -factorizations $F-6$ associated with 13 and $F-13$ associated with 14. Multiplying 1-factor $F-6$ by $7(\bmod 13)$ gives the pairs associated with the point 13 , etc.

The next line of numbers associated with each solution indicates which triple from that orbit is in this solution. The first triple in each orbit is the representative; this is indicated by a " 0 ". Thus solution \#0 consists of the triples $\{1,3,9\},\{2,5,6\},\{4,10,12\},\{8,7,11\},\{1,2,0\},\{5,7,2\}$, $\{9,0,5\},\{6,1,11\}$, etc. The triple $\{0,13,14\}$ is always assumed to be one of the triples in the system.

\section{EXTENSION TO LARGE SETS OF $S Q S(16)$}

Kramer and Mesner [6] enumerated cyclic large sets of $S T S(13)$ and showed that none could be extended to a cyclic large set of $S Q S(14)$. We also checked the solutions enumerated here to see if these could be extended to cyclic large sets of $S Q S(16)$. Only four were found which could possibly be extended. However, none could be. We conclude that no cyclic large set of $S Q S(16)$ exists.

The initial configurations involved the following systems:

$$
\begin{array}{llll}
\text { (1) } & \# 24 & \# 210 & \# 168 \\
\text { (2) } & \# 61 & \# 144 & \# 229 \\
(3) & \# 61 & \# 214 & \# 130 \\
\text { (4) } & \# 61 & \# 214 & \# 255
\end{array}
$$

The initial configurations involved all quadruples containing the fixed points (i.e., $\{13,14,15\})$. Thus we needed three disjoint copies of solutions, one for each fixed point. However, the 1-factors associated with the different systems must agree. Thus, for example, solution \#24 has 1-factors isomorphic to $F-$ 3 and $F-13$; \#210 has 1 -factors isomorphic to $F-3$ and $F-5$; \#168 has 1-factors isomorphic to $F-5$ and $F-13$. In this example we must multiply solution \#168 by $4(\bmod 13)$ to fit it with \#24. To fit \#210 with these two systems, we must multiply by $4 \cdot 8^{-1}(\bmod 13)$ or equivalently by $8 \cdot 3^{-1}(\bmod 13)$. The resulting three systems share these three 1 -factors and must be disjoint as well. 
We also considered potential initial configurations consisting of several isomorphic copies of a solution which possessed an additional automorphism, but none existed. (This case was done with the help of Yeow Meng Chee.)

At this point, one must question whether cyclic large sets of $S Q S(v+3)$ exist. Should this be so, finding a large set of these designs will be very difficult.

\section{BIBLIOGRAPHY}

1. S. Bays, Sur les systèmes cycliques de triples de Steiner différents pour $N$ premier (ou puisance de nombre premier) de la forme $6 n+1$, II-III, Comment. Math. Helv. 3 (1931), 22-41.

2. F. N. Cole, L. D. Cummings, and H. S. White, Complete enumeration of triple systems in 15 elements, Proc. Nat. Acad. Sci. U.S.A. 3 (1917), 197-199.

3. R. H. F. Denniston, Some packings with Steiner triple systems, Discrete Math. 9 (1974), 213-227.

4. __ Sylvester's problems of the 15 school girls, Discrete Math. 9 (1974), 229-233.

5. M. Hall, Jr., and J. D. Swift, Determination of Steiner triple systems of order 15, MTAC 10 (1956), 186-194.

6. E. S. Kramer and D. M. Mesner, The possible (impossible) systems of 11 disjoint $S(2,3,13)$ 's $(S(3,4,14)$ 's) with automorphism of order 11, Utilitas Math. 7 (1975), 55-58.

7. __ Intersections among Steiner systems, J. Combin. Theory Ser. A 16 (1974), 273-285.

8. Jia-Xi Lu, On large sets of disjoint Steiner triple systems. I, J. Combin. Theory Ser. A 34 (1983), 140-146.

9. __, On large sets of disjoint Steiner triple systems. II, J. Combin. Theory Ser. A 34 (1983), 147-155.

10. __ On large sets of disjoint Steiner triple systems. III, J. Combin. Theory Ser. A 34 (1983), 156-182.

11. __, On large sets of disjoint Steiner triple systems. IV, J. Combin. Theory Ser. A 37 (1984), 136-163.

12. __ On large sets of disjoint Steiner triple systems. V, J. Combin. Theory Ser. A 37 (1984), 164-188.

13. $\ldots$, On large sets of disjoint Steiner triple systems. VI, J. Combin. Theory Ser. A 37 (1984), 189-192.

14. __ On large sets of disjoint Steiner triple systems. VII, manuscript.

15. R. A. Mathon, K. T. Phelps, and A. Rosa, Small Steiner systems and their properties, Ars Combin. 15 (1983), 3-100.

16. R. M. Wilson, Some partitions of all triples into Steiner triple systems, Hypergraph Seminar, Lecture Notes in Math., vol. 411, Springer, Berlin, 1974, pp. 267-277.

Department of Algebra, Combinatorics and Analysis, Auburn University, Auburn UNIVERSITY, Alabama 36849-5307

E-mail address: ktphelps@auducvax.bitnet 


\title{
Supplement to Cyclic Large Sets of Steiner Triple Systems of Order 15
}

\author{
By Kevin T. Phelps
}

\begin{tabular}{|c|c|c|}
\hline & $\begin{array}{r}\mathrm{Al} \\
\text { Summ }\end{array}$ & $\begin{array}{l}\text { ENDIX } \\
\text { Listing }\end{array}$ \\
\hline cycle vector & STS & solution number(s) \\
\hline$(0,0,15,90)$ & 80 & \\
\hline$(0,15,11,79)$ & 71 & $134,19,23,29,38,73$ \\
\hline$(0,15,14,76)$ & 69 & $138,146,61,65$ \\
\hline$(0,15,16,74)$ & 67 & $10,135,155,184,91$ \\
\hline$(0,15,16,74)$ & 72 & \\
\hline$(0,18,11,76)$ & 68 & $108,151,213,26,79$ \\
\hline$(0,18,13,74)$ & 78 & 43 \\
\hline$(0,18,14,73)$ & 66 & $223,227,238,241,252,9,94$ \\
\hline$(0,18,21,66)$ & 73 & \\
\hline$(0,18,9,78)$ & 79 & 0,2 \\
\hline$(0,21,14,70)$ & 65 & $118,148,166,215,235,51,96$ \\
\hline$(0,21,15,69)$ & 75 & 211,74 \\
\hline$(0,21,7,77)$ & 62 & $167,183,27$ \\
\hline$(0,21,7,77)$ & 63 & 30 \\
\hline$(0,24,7,74)$ & 74 & \\
\hline$(0,27,12,66)$ & 70 & $123,147,163,177,206,48,53,85$ \\
\hline$(0,30,10,65)$ & 64 & $170,217,45,76$ \\
\hline$(0,30,5,70)$ & 76 & 161,246 \\
\hline$(0,42,7,56)$ & $61^{*}$ & 111 \\
\hline$(0,6,15,84)$ & 77 & $113,186,59$ \\
\hline$(1,12,11,81)$ & 57 & $127,185,191,250,50,83,98$ \\
\hline$(1,15,12,77)$ & 50 & $110,21,49,87$ \\
\hline$(1,18,12,74)$ & 49 & $126,197,4$ \\
\hline$(1,18,14,72)$ & 46 & $139,212,32,40,92$ \\
\hline$(1,18,18,68)$ & 60 & $103,124,181,60$ \\
\hline$(1,21,10,73)$ & 48 & $114,169,172,180,42,6,68$ \\
\hline$(1,21,12,71)$ & 56 & $154,158,17,173,176,229,62,90$ \\
\hline$(1,21,20,63)$ & 42 & $106,16,216,244,67,70,97$ \\
\hline$(1,21,7,76)$ & 58 & $101,145,160,192,224,225,226,36,72,75$ \\
\hline$(1,21,8,75)$ & 44 & 115 \\
\hline$(1,24,12,68)$ & 45 & 190,41 \\
\hline$(1,24,12,68)$ & 52 & $100,104,140,200,205,249,34,58$ \\
\hline$(1,24,15,65)$ & 55 & $11,116,44$ \\
\hline$(1,27,9,68)$ & 47 & $157,162,187,198$ \\
\hline$(1,33,8,63)$ & 33 & $12,156,189,20,232,236,242$ \\
\hline$(1,36,16,52)$ & 59 & $136,143,240,81$ \\
\hline$(1,36,9,59)$ & 32 & $174,66,86$ \\
\hline$(2,12,12,79)$ & 37 & 1, \\
\hline$(2,21,13,69)$ & 38 & $131,175,239,24$ \\
\hline$(2,21,15,67)$ & 51 & $121,203,248,28,3,39,69$ \\
\hline$(2,24,4,75)$ & 36 & 220,71 \\
\hline$(2,24,9,70)$ & 53 & $109,178,209,230,233,54$ \\
\hline
\end{tabular}

(C) 1990 American Mathematical Society $0025-5718 / 90 \$ 1.00+\$ .25$ per page 

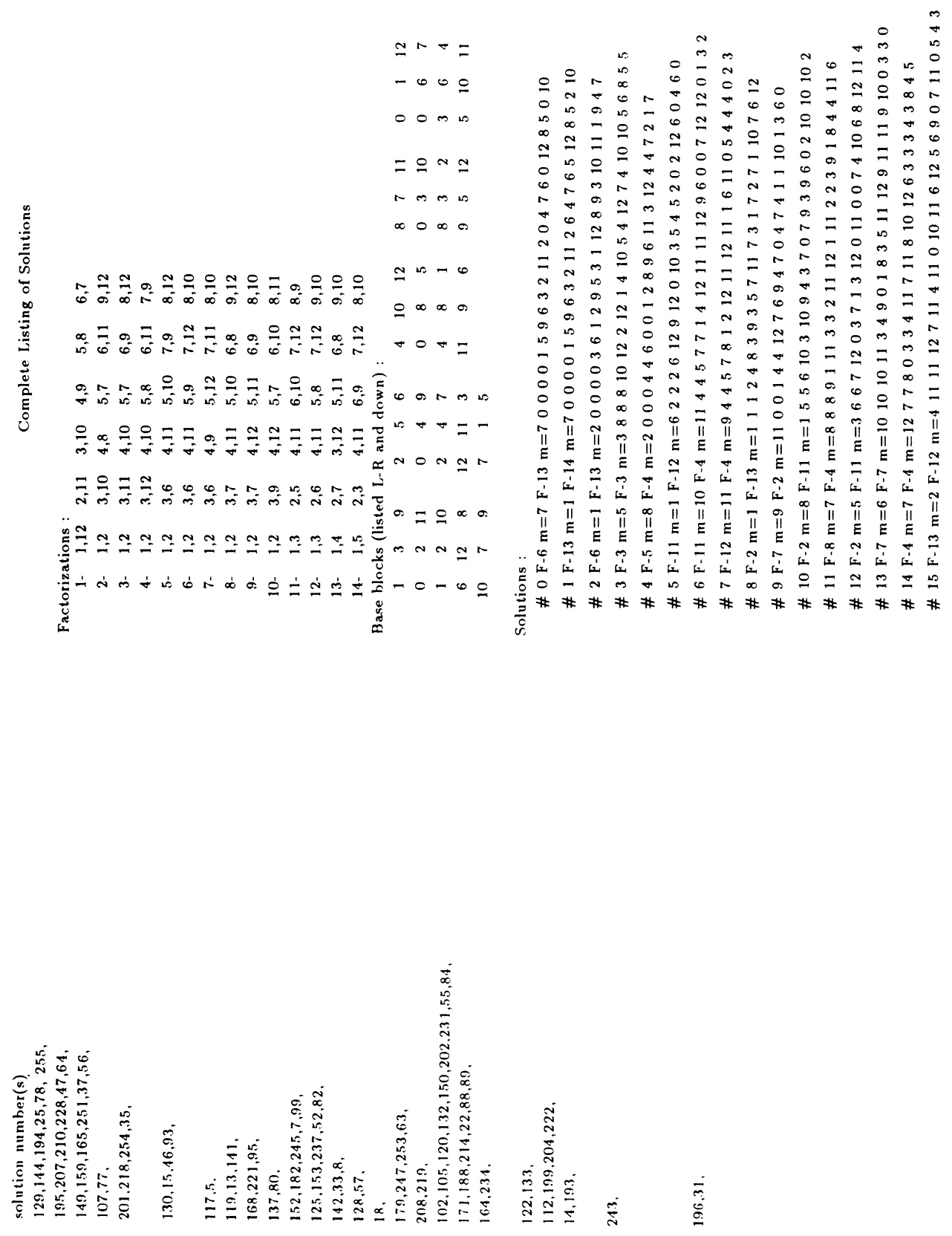

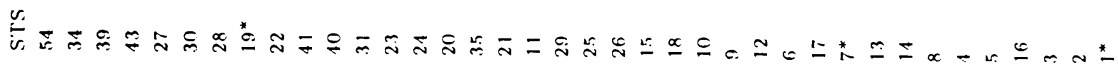

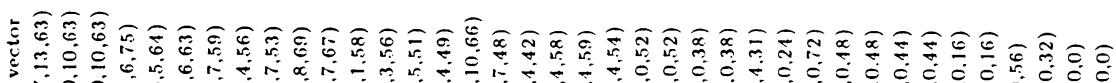

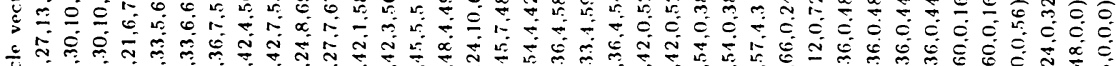

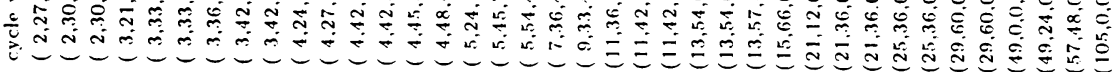




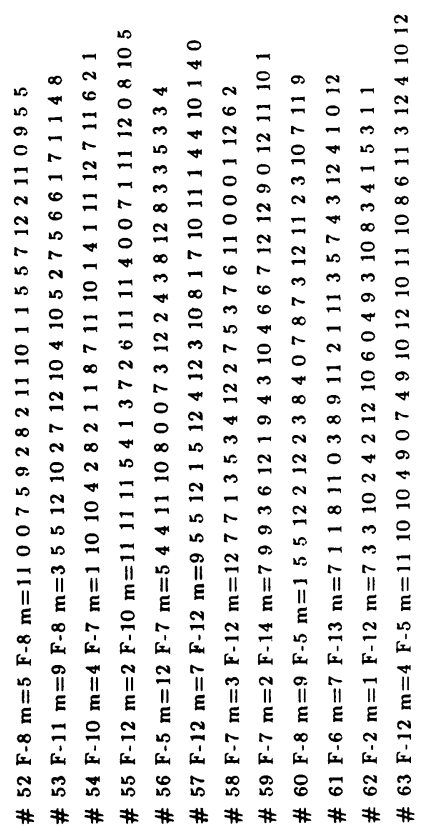

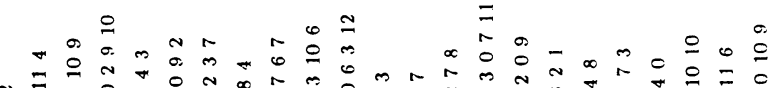

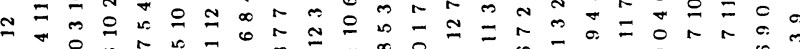

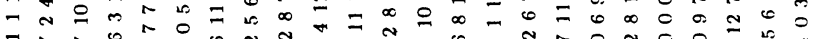

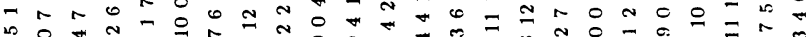

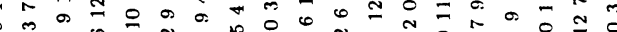

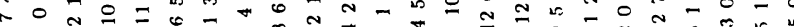

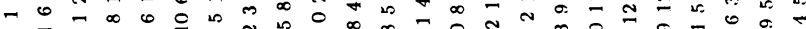

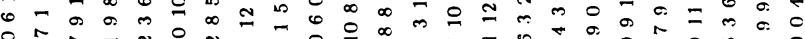

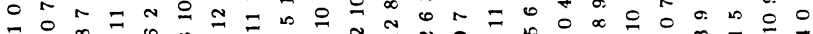

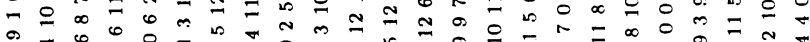

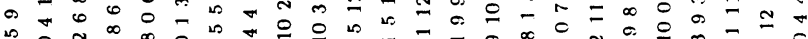

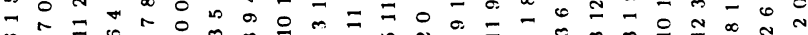

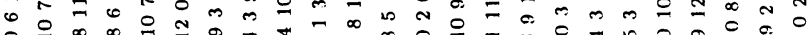

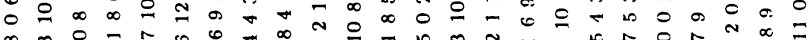

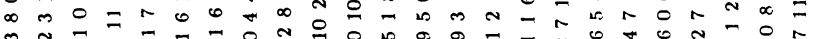

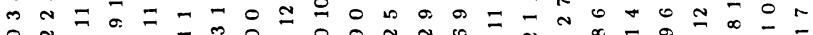

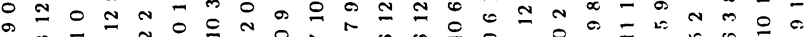

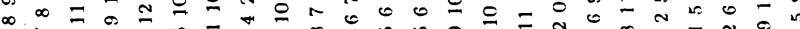

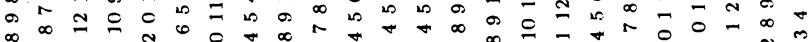

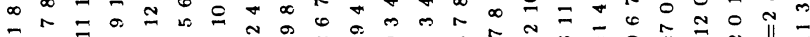

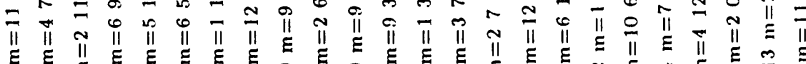
E $E$ E $\cong$ ก

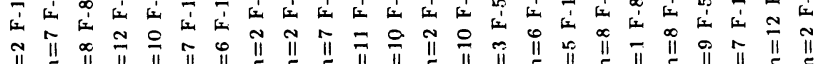

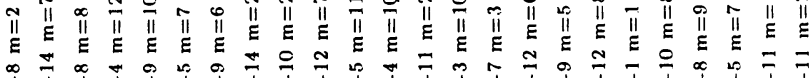

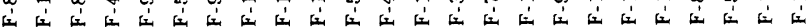

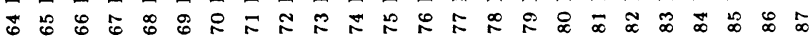

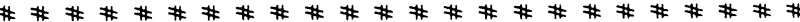




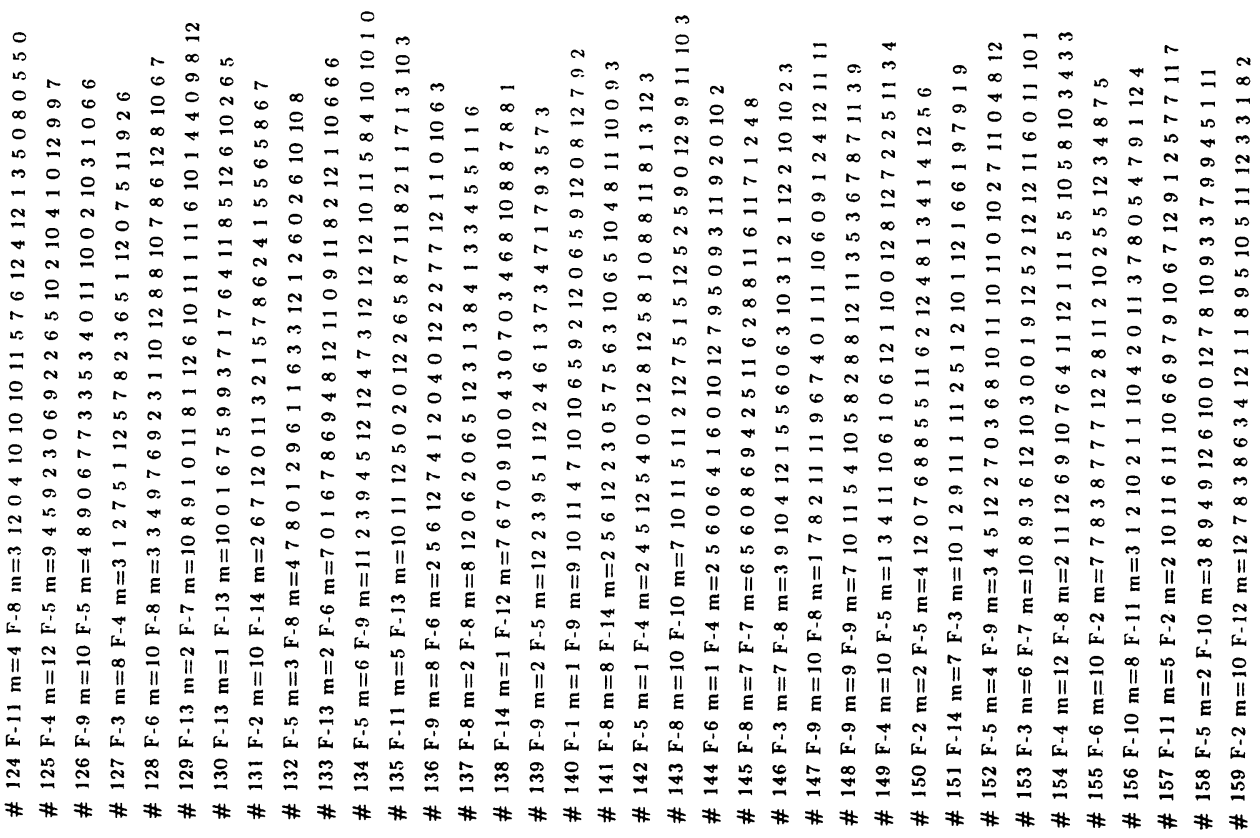

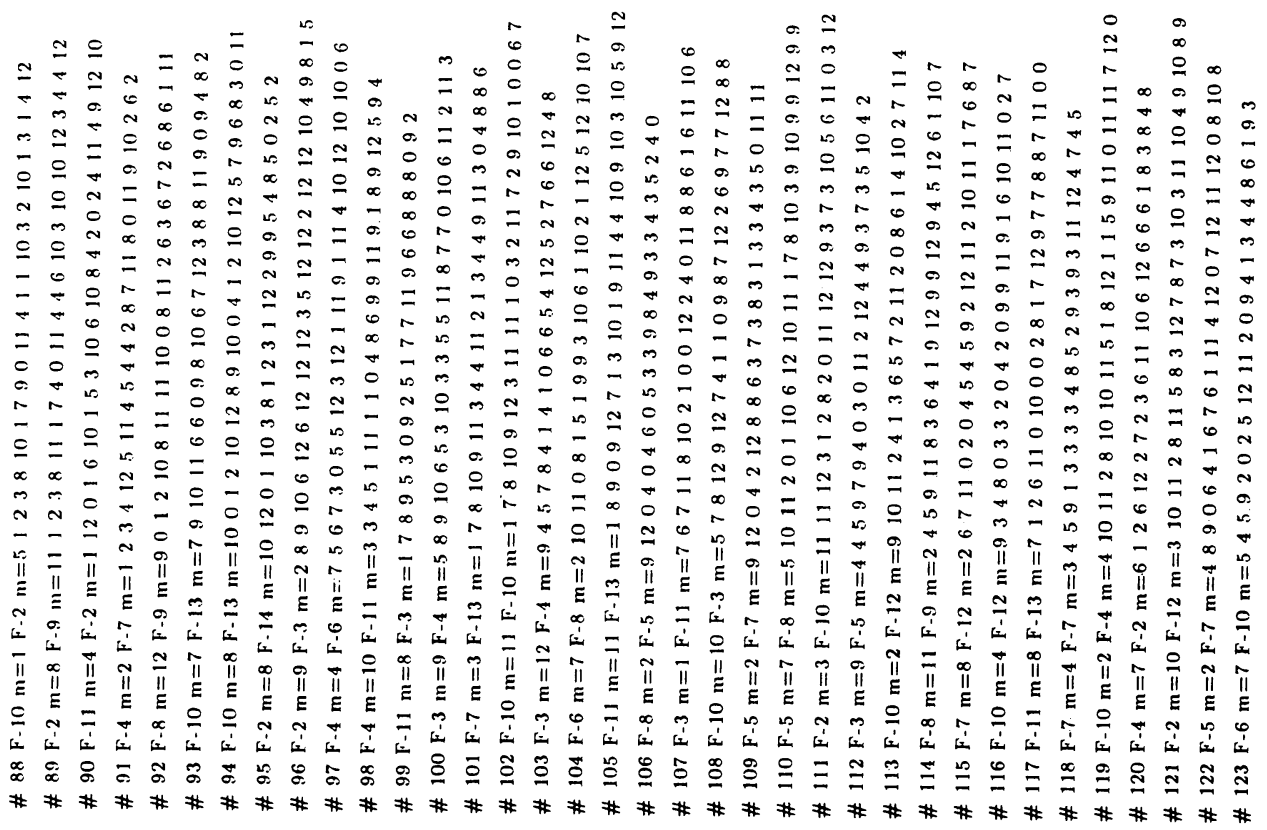




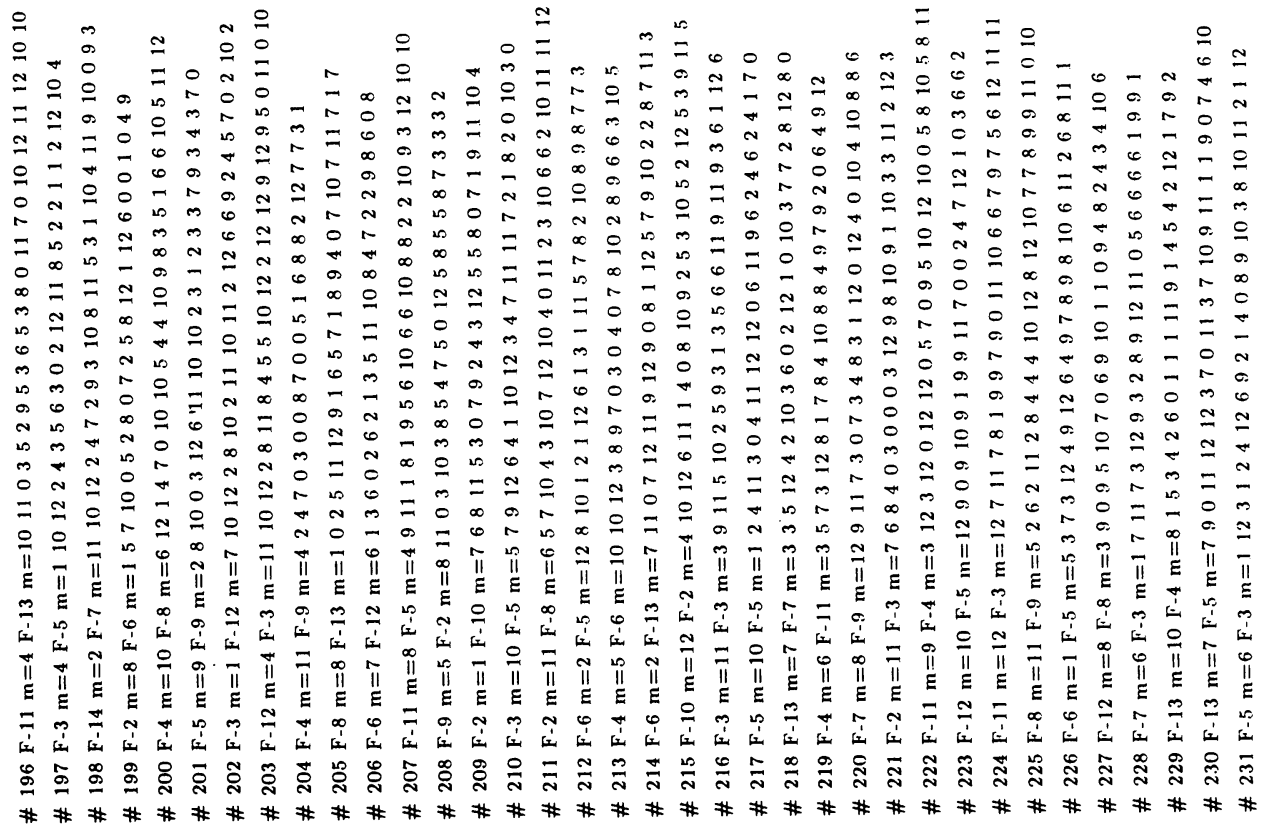

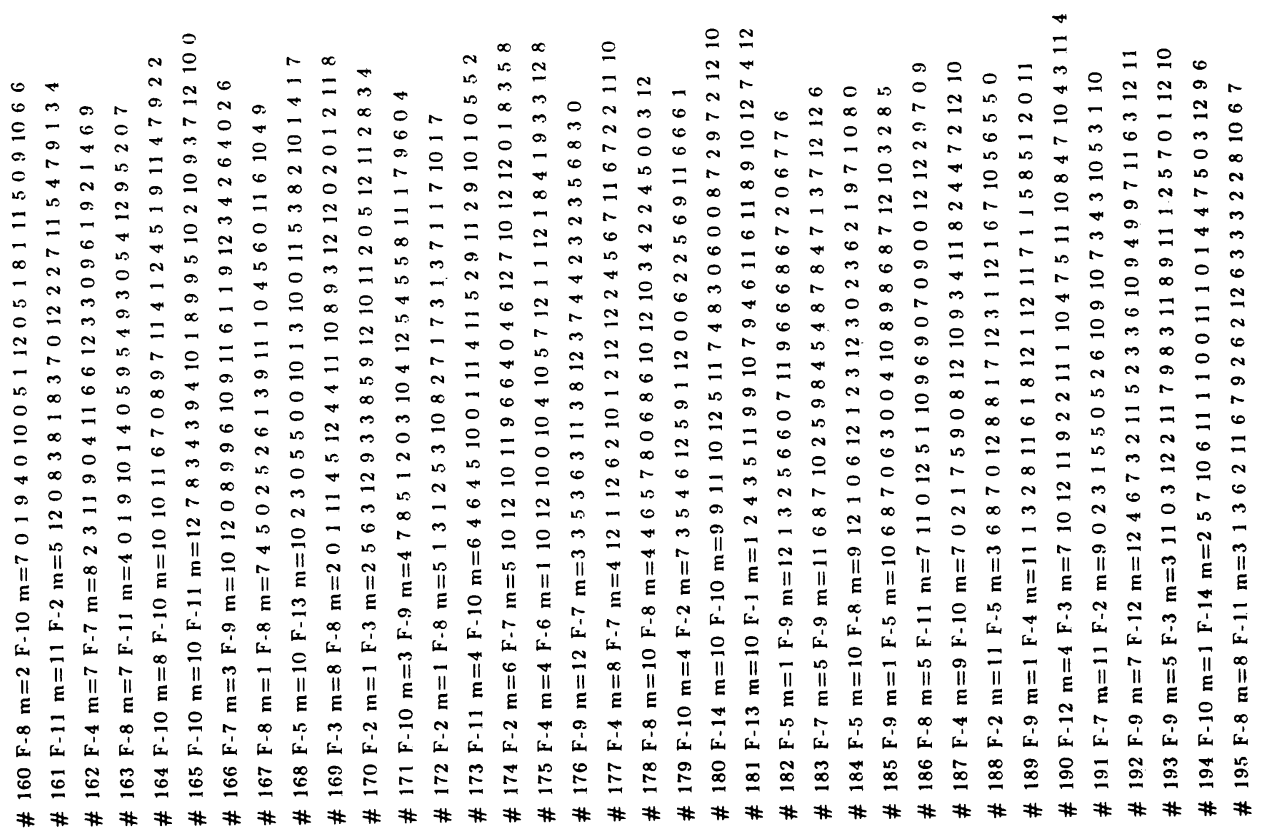




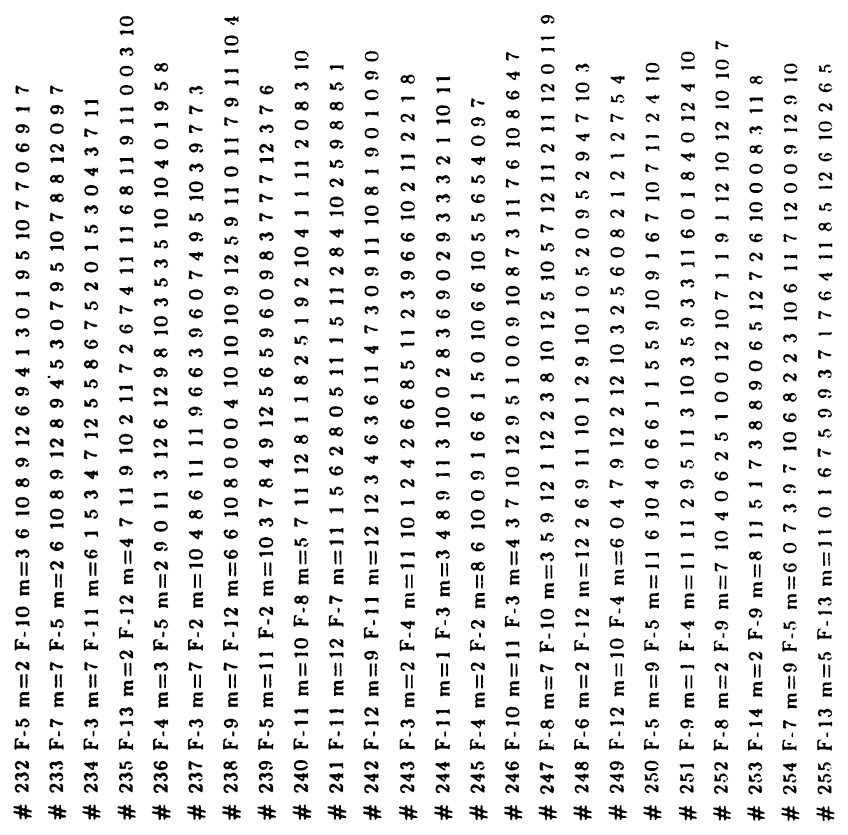

